\title{
San Paolo secondo Pasolini: ascesi e organizzazione
}

\author{
Silvia Giuliani
}

\section{OpenEdition}

\section{Journals}

Edizione digitale

URL: http://journals.openedition.org/cei/196

DOI: $10.4000 /$ cei. 196

ISSN: 2260-779X

Editore

UGA Éditions/Université Grenoble Alpes

\section{Edizione cartacea}

Data di pubblicazione: 15 juillet 2009

Paginazione: $115-125$

ISBN: 978-2-84310-145-8

ISSN: $1770-9571$

Notizia bibliografica digitale

Silvia Giuliani, «San Paolo secondo Pasolini: ascesi e organizzazione», Cahiers d'études italiennes [Online], 9 | 2009, online dal 15 janvier 2011, consultato il 26 mars 2021. URL: http:// journals.openedition.org/cei/196 ; DOI: https://doi.org/10.4000/cei.196 


\title{
SAN PAOLO SECONDO PASOLINI: ASCESI E ORGANIZZAZIONE
}

\author{
Silvia Giuliani \\ EPHE Paris e Università di Pisa
}

La categoria del sacro costituisce una costante intrinseca ${ }^{\mathrm{I}}$ della visione del mondo pasoliniana ed estrinseca (intesa cioè come contenuto o messaggio di un'opera) della produzione dell'autore fin dai suoi esordi, categoria che si dipana attraverso una complessa fenomenologia e che, se letta a 360 gradi, fornisce un'importante cartina tornasole della situazione sociale coeva e dell'approccio dell'autore a essa.

Già nella raccolta poetica Le ceneri di Gramsci il sacro compare a definire forme di vita barbare, impure: "Più è sacro dove più è animale il mondo" (Pasolini, 1975, pp. 43-51 ${ }^{2}$ ); spesso si rivela ai margini della normalità, nelle zone di periferia, nei "rifiuti del mondo" e si manifesta nel senso di stupore e di mistero che appartiene a culture escluse dal progresso, finendo per rappresentare ciò che si oppone alla mercificazione del mondo capitalista e borghese.

La lettura del Trattato di storia delle religioni di Mircea Eliade (Eliade, 1948) contribuisce, nella percezione di Pasolini, a radicare in modo ancora più saldo il sacro al mondo atavico contadino caratterizzato da una visione del tempo ciclica legata alla morte e alla rinascita, al raccolto e alla semina. In Medea la sacralità è rappresentata dai riti e culti barbarici legati ancora una volta alla fertilità da propiziarsi, se occorre, con sacrifici umani.

Ma soprattutto Pasolini riprende da Eliade il concetto di ierofania, come apparizione e mostrarsi del sacro nella natura in una sorta di immanenza, e scopre "l'immensità del mondo arcaico contadino" e "la matrice

\footnotetext{
I. "Io sono ateo, ma il mio rapporto con le cose è pieno di mistero e di sacro. Per me niente è naturale nemmeno la natura." (Da un'intervista a La Stampa del I2 luglio i968)

2. I riferimenti bibliografici forniti in corpo testo in forma abbreviata si trovano sciolti nell'elenco bibliografico di fine articolo.
} 
cosmogonica della sua religione" (Conti Calabrese, I994, p. Io). Tale concetto è legato alla natura friulana con cui il poeta è a contatto nella sua giovinezza e al suo primo immergersi in essa.

Larga parte della produzione successiva dell'autore sarà dedicata alla ricerca del sacro ormai annullato dalla nuova cultura industriale che fagocita e livella ogni esperienza "altra".

È proprio alla fine degli anni Sessanta che i due poli della questione si radicalizzano: il neocapitalismo e l'omologazione avanzano inesorabilmente; la seconda rivoluzione industriale e l'impero della tecnologia hanno ormai preso piede ${ }^{3} \mathrm{e}$ in maniera inversamente proporzionale a essi si pone la richiesta e la comparsa del sacro in una società siffatta. L'inferno neocapitalista rischia di diventare "la storia di una originaria rimozione della sacralità del sacro" (Conti Calabrese, I994, p. I8), fagocitata dalla "sacralità dei consumi" e delle merci come feticci, storia di cui Salò diventerà in seguito la più sconcertante e "scandalosa" metafora cinematografica.

Il problema che inquieta Pasolini è come in un'epoca così profondamente desacralizzata, nell' "Ordine Orrendo", si possa ancora enunciare il sacro e l'enucleazione del problema diventa per lui tutt'uno, come si accennava, con l'ergersi a strenuo difensore di questo: "Io difendo il sacro perché è la parte dell'uomo che offre meno resistenza alla profanazione del potere, ed è la più minacciata dalle istituzioni delle Chiese." (Duflot, I970, p. 78)

Prima di addentrarci più a fondo nella riflessione di Pasolini sul sacro è necessario soffermarsi proprio sulla parola chiave "Chiesa" e chiarire la percezione che l'autore ha di questa istituzione. Parafrasando o citando stralci delle riflessioni pasoliniane diffuse qua e là negli Scritti Corsari (Pasolini, I975b, pp. I4-I7, 66-69) si può affermare che in passato la Chiesa è scesa a patti col diavolo facendosi strumento repressivo del potere: Dio, Patria, Famiglia, Virtù civili e familiari erano solo i pretesti dietro i quali il vecchio potere agiva indisturbato; ma il nuovo Potere non ha più bisogno di quei pretesti, ma solo di adepti del nuovo culto delle merci e di "un universo tecnicistico e puramente terreno in cui possa svolgersi secondo la propria natura il ciclo della produzione e del consumo"; la Chiesa, divenuta inutile al Potere, sta dunque per essere accantonata (insieme al sacro), dimenticata dal mondo, proprio come ammette lo stesso Paolo VI in un discorso di contrizione e ammissione di responsabilità dell'istitu-

3. Si vedano a tal proposito gli articoli polemici e sferzanti che appaiono nelle testate con cui collabora in questo momento Pasolini, Il Corriere della Sera e Il Mondo, che confluiranno poi nelle Lettere luterane e negli Scritti corsari. 
zione Chiesa. Questo il rimedio che l'autore propone per arginare la difficile situazione:

In una prospettiva radicale, forse utopistica, o, è il caso di dirlo, millenaristica, è chiaro [...] ciò che la Chiesa dovrebbe fare per evitare una fine ingloriosa. Essa dovrebbe passare all'opposizione. [...] Dovrebbe passare all'opposizione contro un potere che l'ha così cinicamente abbandonata, progettando, senza tante storie, di ridurla a puro folclore. Dovrebbe negare se stessa, per riconquistare i fedeli (o coloro che hanno un "nuovo" bisogno di fede) che proprio per quello che essa è l'hanno abbandonata. (Pasolini, 1975b, p. 68)

$\grave{\mathrm{E}}$ in questo contesto socio-politico-culturale che si colloca la sceneggiatura per un film dedicato a san Paolo, organizzata nella sua fase embrionale in materiale letterario, nella forma di appunti scritti di getto tra il maggio e il giugno 1968; la produzione fu inizialmente affidata alla Sanpaolo Film, ma a causa delle perplessità di don Cordero, allora direttore della compagnia, il progetto si arenò. Venne ripreso nel 1974 e ritoccato da Pasolini in vista della realizzazione da parte di nuovi produttori, ma anche in questo caso il progetto non ebbe successo. Il materiale relativo (sul quale si basa il presente lavoro), composto da un Progetto e un Abbozzo di sceneggiatura, è uscito postumo per i tipi di Einaudi nel $1977 \mathrm{col}$ titolo di San Paolo.

Alla domanda posta da Bachmann in un'intervista del 1974 sui motivi della realizzazione del film, Pasolini risponde riportando ai nostri occhi tutta l'urgenza di una polemica dura, ma nello stesso tempo parenetica, nei confronti della situazione della Chiesa. Ecco dunque una tra le svariate dichiarazioni (spesso anche in contrasto tra loro secondo lo stile dell'autore) che ci fornisce indicazioni su come interpretare questo scritto singolare:

Lo faccio - proseguiva Pasolini - proprio perché il Papa Paolo ha fatto un discorso di contrizione terribile $[. .$.$] . Dice chiaramente che ormai la società non ha più bisogno$ della Chiesa, la società provvede da se stessa ai suoi bisogni; e quindi dove interviene la Chiesa? Quali sono gli interventi che la Chiesa può fare in favore di qualcuno? [...] La conclusione, poi, è misera: cioè, come ovviare a tutto questo? Pregando. E va bene, ma chi prega se la situazione si è messa in modo tale che nessuno prega più? Allora quello che mi fa rabbia è questo: ora che il potere borghese la esclude con tanto cinismo dopo essersi appoggiato su di lei per un secolo; ora che il potere borghese, dopo che l'ha sfruttata per tanti anni, non sa più che farsene e l'ha buttata a mare, la Chiesa dovrebbe cambiare radicalmente politica e passare decisamente all'opposizione. A questo punto, diventata religione vera, dovrebbe opporsi con violenza al potere, e invece non lo fa: allora, se mi ha fatto rabbia che per tanti secoli fosse asservita al potere, fosse potere essa stessa, mi fa più rabbia ora che tace in un momento in cui dovrebbe parlare, dovrebbe mettersi alla testa dell'opposizione, perché l'opposizione al nuovo potere non può essere che un'opposizione di carattere anche religioso [...]. Anche il Papa non dovrebbe esserci più; la Chiesa dovrebbe rinnovarsi completamente, negarsi se vuol chiamarsi Chiesa nel senso etimologico della 
parola "ecclesia", cioè adunanza di gente e non gestione dall'alto del Vaticano... (De Giusti, 1974, pp. 156-157)

Il materiale su san Paolo appare dunque da principio strettamente legato alla polemica contro la Chiesa portata avanti anche negli Scritti corsari.

Nonostante tutto Pasolini riconosce nella Chiesa, in quanto comunità organizzata di fedeli, l'eredità di un sentimento del sacro che, se pur dimenticato e fagocitato da altri valori, custodisce ancora il significato della sua origine e la chiave del suo destino. Affinché però al suo interno possa riemergere un rinnovato senso religioso è necessario che questa si neghi in quanto gerarchia teocratica e secolarizzata, unico modo per conservarsi intatta nei continui compromessi con i diversi sistemi di potere avvicendatisi nel corso dei secoli.

Procediamo ora con l'analisi diretta dello scritto e con le preliminari dichiarazioni dell'autore prima di trarre qualsiasi conclusione e verificare (o forse scoprire?) dove conduce la strategia del testo.

La novità del racconto cinematografico di Pasolini sta nella trasposizione della storia di san Paolo nel presente. In tale prospettiva "il mondo in cui vive e opera San Paolo è dunque quello del 1966 o 1967: di conseguenza è chiaro che tutta la toponomastica deve essere spostata" (Pasolini, I977, p. 6) ${ }^{4}$. San Paolo, il fariseo (di famiglia romano-giudaica), è rappresentato come un borghese la cui caratteristica è di vivere in uno stato di "inconsapevole insincerità". Le sue vicende cominciano sulla via di Damasco (nella versione pasoliniana Barcellona), la città dove si sono rifugiati Pietro e i suoi fedeli e dove Paolo chiede di continuare la sua persecuzione contro i cristiani. Per raggiungerla deve però attraversare il deserto, cioè le strade dell'Europa, e proprio in una di queste strade, "piene di traffico e dei soliti atti della vita quotidiana, ma perdute nel silenzio, Paolo è colto dalla luce. Cade, e sente la voce della vocazione" (Pasolini, I977, p. 9). Così, dopo aver raggiunto Barcellona ed essersi unito agli altri cristiani, dà avvio all'“avventura della predicazione", che si svolge in Italia e in particolare nella "moderna Roma, scettica, ironica, liberale". Dall'Italia il suo viaggio prosegue poi verso nord dove avviene un'altra trasposizione: il sogno del Macedone diventa il sogno di un Tedesco che appare a Paolo e lo supplica, elencando tutti i problemi reali e le degradazioni morali che travagliano il paese, di mettersi in viaggio per la Germania. Paolo ubbi-

4. In realtà all'inizio dell' $A b b o z z o$ l'autore dichiara che l'antica Gerusalemme è sostituita da Parigi negli anni tra il ' 38 e il ' 44 , cioè sotto l'occupazione nazista. Si può dunque approssimare un lasso di tempo entro il quale si collocano la vita e le vicende di Paolo, cioè tra gli anni '30 e metà anni '70; d'altra parte anche gli Atti degli Apostoli prevedono che il racconto si collochi in un certo arco temporale, all'incirca tra il 30 e il 63 d.C. 
disce e si avvia "col passo veloce e sicuro del Santo, lungo una immensa autostrada che porta verso il cuore della Germania..." (Pasolini, I977, p. II). Arrestato, Paolo viene processato a Vichy, in luogo di Cesarea, ma chiede di essere giudicato a Roma, che diventa nella concezione pasoliniana la New York contemporanea. Così si esprime Pasolini per dare credibilità alla sua scelta: "Lo stato d'ingiustizia dominante in una società schiavista come quella della Roma imperiale può essere qui adombrato dal razzismo e dalla condizione dei negri." Di fatto

è il mondo della potenza, della ricchezza immensa dei monopoli, da una parte, e dall'altra dell'angoscia, della volontà di morire, della lotta disperata dei negri, che San Paolo si trova ad evangelizzare. E quanto più "santa" è la sua risposta, tanto più essa sconvolge, contraddice e modifica la realtà attuale (Pasolini, 1977, p. 12).

San Paolo viene rinchiuso infine in un carcere americano; condannato a morte, subisce il martirio nella strada di una metropoli, dove la gente passa e rimane indifferente "allo spettacolo della morte". La rivoluzione di Paolo è frutto della sua "Parola"; ne consegue che, nonostante il cambio di scenario, Pasolini voglia che il centro di quella "santità" veicolata attraverso la parola rimanga integro: "Come ho già fatto per il Vangelo, nessuna delle parole usate da Paolo nel dialogo del film sarà inventata o ricostruita per analogia" (Pasolini, I977, p. 5). Il lavoro risulta infatti costellato di citazioni tratte dalle lettere del Santo (e in minor misura anche dagli Atti degli Apostoli), inserite immodificate in un contesto moderno. Continua l'autore nel Progetto:

Le "domande" che gli evangelizzati porranno a San Paolo saranno domande di uomini moderni, specifiche, circonstanziate, problematiche, politiche, formulate con un linguaggio tipico dei nostri giorni; le "risposte" di San Paolo, invece, saranno quelle che sono: cioè esclusivamente religiose, e per di più formulate col linguaggio tipico di San Paolo, universale ed eterno, ma inattuale (in senso stretto). Così il film rivelerà attraverso questo processo la sua profonda tematica: che è contrapposizione di "attualită" e di "santită" - il mondo della storia, che tende, nel suo eccesso di presenza e di urgenza, a sfuggire nel mistero, nell'astrattezza, nel puro interrogativo - e il mondo del divino, che nella sua religiosa astrattezza, al contrario, discende tra gli uomini, si fa concreto e operante. (Pasolini, 1977, p. 7)

Scendendo nei dettagli, il "plot narrativo" presenta una macrocontrapposizione tra due filoni che corrono paralleli e che vedono rispettivamente come protagonista il Paolo santo e il Paolo prete, ex-fariseo e fondatore della Chiesa cattolica (De Giusti, 1974, p. 159)

5. Queste le parole di Pasolini in proposito nella citata intervista (De Giusti, 1974, p. I59): "Il film è una cosa violentissima contro la Chiesa e contro il Vaticano, perché faccio un san Paolo doppio, cioè schizofrenico, 
Il primo nella sua esperienza ascetico mistica, dall'accecamento alla meditazione nel deserto, dal martirio fino al "rapimento al Terzo Cielo", viene descritto come creatura umile e malata, tormentata dal problema di Dio e dai patimenti che soffre in suo nome; a lui si lega la parte statica della trama: è solo a riflettere tra dolori fisici e morali nello studio di una casa borghese o in una stanza d'albergo. A lui vanno chiaramente le simpatie dell'autore. Il secondo, l'ex-fariseo (lo scritto si apre sul volto cinico di un Paolo che assiste impietoso al massacro di Stefano) è il Paolo che in una sequela di viaggi organizzativi, elettorali o catechistici si impegna a dettare le norme e le leggi alle nuove comunità cristiane: forte, sicuro di sé, fanatico fino all'antipatia.

L'attenzione dell'autore si concentra particolarmente proprio sul Paolo "prete" e sulle tappe che hanno condotto alla formazione della Chiesa come istituzione, in un cammino a ritroso che ne mostri gli errori e le tare ataviche già tutte presenti, secondo l'autore, a questa altezza cronologica: la sessuofobia, l'antifemminismo, l'organizzazione, le collette, il trionfalismo, il moralismo. Da un certo punto del racconto in poi il Santo viene del tutto perduto di vista; la funzione dell'apostolo è sostituita da quella di un "portaparola" che ripete idee sulla non inconciliabilità di carisma e istituzione. Si prendano a titolo di esempio alcuni passi della predicazione di Paolo a Genova (Corinto) in casa dell'adepta Priscilla, rivolta soprattutto ai non eletti, ai Gentili, che nelle intenzioni dell'autore corrisponderebbero nella modernità a una borghesia laica, liberale e materialista che ospita nel suo alveo molti intellettuali; proprio i commenti scandalizzati, critici e disincantati di costoro (rivolti oltre che al moralismo e fanatismo di Paolo, anche al suo essere "organizzatore") dovevano costituire nelle intenzioni originarie un controcanto continuo alla predicazione di Paolo. Ecco le parole di uno degli apostoli durante la predicazione a Parigi:

Il nostro è un movimento organizzato... Partito, Chiesa... chiamalo come vuoi. Si sono stabilite delle istituzioni anche fra noi, che contro le istituzioni abbiamo lottato e lottiamo. L'opposizione è un limbo. Ma in questo limbo già si prefigurano le norme che faranno della nostra opposizione una forza che prende il potere: e come tale sarà un bene di tutti. Dobbiamo difendere questo futuro bene di tutti, accettando, sì, anche di essere diplomatici, abili, ufficiali. Accettando di tacere su cose che si dovrebbero dire, di non fare cose che si dovrebbero fare, o di fare cose che non si dovrebbero

nettamente dissociato in due: uno è il santo [...] l'altro invece è il prete, ex-fariseo, che recupera le sue situazioni culturali precedenti e che sarà il fondatore della Chiesa. Come tale lo condanno; come mistico va bene, è un'esperienza mistica come altre, rispettabile, non la giudico, e invece lo condanno violentemente come fondatore della Chiesa, con tutti gli elementi negativi della Chiesa già pronti: la sessuofobia, l'antifemminismo, l'organizzazione, le collette, il trionfalismo, il moralismo. Insomma tutte le cose che hanno fatto il male della Chiesa sono già tutte in lui." 
fare. Non dire, accennare, alludere. Essere furbi. Essere ipocriti. Fingere di non vedere le vecchie abitudini che risorgono in noi e nei nostri seguaci - il vecchio ineliminabile uomo, meschino, mediocre, rassegnato al meno peggio, bisognoso di affermazioni e di convenzioni rassicuranti. Perché noi non siamo una redenzione, ma una promessa di redenzione. Noi stiamo fondando una Chiesa. (Pasolini, 1977, p. II4)

Agli occhi degli intellettuali Paolo "crea una legge e istituisce una Chiesa. É, soprattutto, un grande organizzatore. Metà mondo è ormai costellato dai suoi centri organizzativi. [...] È un uomo estremamente pratico, un burocrate, un generale" (Pasolini, 1977, p. 95). E ancora:

Egli è fondatore di Chiese: ha l'ossessione dell'istituire. [...] Questo è niente: possibile piuttosto, che egli venga a predicare una chiesa clericale là dove, se si sente il bisogno di una chiesa, questa non può essere che ecumenica: e che se qualcosa essa deve insegnare, in linea di principio, non può essere che la resistenza all'autorità, a ogni genere di autorità? (Pasolini, 1977, p. I42)

A proposito di istituzioni c'è un altro interessante nucleo della trama che ci aiuta a capire la preferenza di Pasolini per il versante del "trasumanar" della figura paolina piuttosto che di quello dell'“organizzar" (il dilemma alla base della raccolta poetica del I97I si ripropone in nuove forme nella figura di Paolo, che non a caso come vedremo è anche citata a più riprese nella raccolta) ed è costituito dalle sezioni di testo spesso in corsivo (aggiunte durante i rimaneggiamenti del '74) che descrivono l'impossessamento da parte di Satana dell'evangelista Luca affinché egli descriva negli Atti degli Apostoli l'organizzazione ecclesiastica che Paolo sta creando in modo da mettere in rilievo soprattutto gli aspetti più istituzionali e burocratici dell'operazione di Paolo, e nello stesso tempo l'altra faccia della fede, il potere temporale della Chiesa. Paolo, strumento operativo nelle mani di Satana, ha compiuto la sua volontaria-involontaria missione, ha fondato la Chiesa dotandola in potenza di tutte le efferatezze e gli abusi che non tarderà a perpetrare. Che Paolo ascenda pure gloriosamente al Terzo Cielo, ormai il suo dovere è compiuto:

Egli [Satana] entra nel palazzo e sale all'appartamento di Luca. [...] Luca riassume ghignando al suo capo la continuazione della storia di Paolo. Praticamente ormai il fine è raggiunto. La Chiesa è fondata. Il resto non è che una lunga appendice, un'agonia. A Satana non interessa il destino di Paolo: si salvi pure e se ne vada pure in Paradiso. Satana e il suo sicario sghignazzano soddisfatti. Luca si alza, prende da un mobiletto dello champagne e i due brindano ripetutamente alla loro Chiesa. Bevono e si ubriacano, evocando tutti i delitti della Chiesa: elenco lunghissimo di papi criminali, di compromessi della Chiesa col potere, di soprusi, violenze, repressioni, ignoranza, dogmi. Alla fine i due sono completamente ubriachi e ridono pensando a Paolo che è ancora là, in giro per il mondo a predicare e organizzare. (Pasolini, I977, pp. I43-I44) 
Delineata con chiarezza la pars destruens del testo, nel percorso a ritroso verso la fondazione della Chiesa come istituzione, futura complice e detentrice del potere temporale, denunciati ab ovo i momenti che hanno portato all'attualità deprecata da Pasolini e condannato senza attenuanti il momento dell'“organizzar", è però possibile individuare una pars construens che Pasolini extratestualmente suggerisce tramite la figura del Santo e la sua "parola" più duratura: si tratta di quella individuabile nella carità, concetto chiave della meditazione pasoliniana sulla Chiesa e nello stesso tempo marca principale della predicazione paolina.

Pasolini sembra infatti pensare a una ecclesia che attraverso la carità non solo possa riscoprire l'originario spirito di amore fraterno, ma anche quel particolare sentimento del sacro che solo potrebbe portarla a ripristinare (o più probabilmente a generare) costumi e consuetudini capaci di sottrarla e di renderla tenace avversaria del nuovo universo capitalistico; la nuova prassi di vita (rituale o meno) che sia in grado di mantenere desto il rapporto sacro e mistico con la natura e con le cose non implica però di fatto la fondazione ex novo di un credo religioso basato su nuovi principi.

È possibile che nelle nuove comunità riformate a partire dalla carità si avverta progressivamente il bisogno di istituire e organizzare, ma sarà certo un altro tipo di organizzazione che possa finalmente unificare in una sintesi che non annulla i contrari, ma li mantiene uno accanto all'altro nella loro identità, il trasumanar e l'organizzar. È significativo a questo proposito il fatto che la compresenza e il compromesso tra l'ascetismo e l'organizzazione (sbilanciato in negativo verso l'“organizzar" di san Paolo) si ritrovi già nel I97I nella poesia Trasumanar e organizzar da cui prende il titolo la raccolta: nel momento in cui Pasolini pensa di iscriversi al PCI e di unire il suo idealismo e la sua battaglia di idee poetico-giornalistica con la prassi di un partito "macchiato di retorica e volgarità", è consapevole della dissociazione "schizoide" cui va incontro, che non poteva non fargli pensare alla vita del Santo:

Eh, è naturale che avrei dovuto poi adattarmi a questa dissociazione.

Ogni calcolo la implica; ogni patto, ogni degradazione:

sarò diviso: tacitato e ufficiale, nell'agire, critico e solo

nello scrivere poesie. Non è questa separazione

che si è sempre voluta - forse giustamente?

Non a caso ho sulla schiena la mano sacra e untuosa di San Paolo che mi spinge a questo passo.

La contemporaneità temporale del trasumanar non è l'organizzar? [...] 
Ma il nostro mondo è schizoide, cari amici, caro funzionario del PCI, a cui è rivolta questa lettera non formale.

(Pasolini, I975, p. 623)

Per concludere sul tema della carità, base d'appoggio per un ritorno del sacro nella contemporaneità su cui Pasolini insiste molto in questo periodo e negli anni precedenti anche attraverso la produzione poetica e la libellistica polemica sui giornali, si possono citare stralci dalla sezione Poesie su commissione, specie il componimento L'enigma di Pio XII (Pasolini, I975, p. 56I $)^{6}$ e sicuramente tra gli Scritti corsari un testo del marzo 1974, Vuoto di carità, vuoto di cultura: un linguaggio senza origini (Pasolini, I975b, pp. 3I-34); infine un articolo comparso nel 1968 sul Tempo nel quale l'autore invita la Chiesa a uno scisma citandone proprio il più attivo fondatore, Paolo di Tarso, di cui riporta una frase della Prima lettera ai Corinti:

Ricorrerò a San Paolo. Nella Prima lettera ai Corinti, si legge questa stupenda frase (non tutto in Paolo è stupendo, spesso parla in lui il prete, il fariseo): "Restano fede, speranza e carità, queste tre cose: di tutte la migliore è la carità."

La carità - questa "cosa" misteriosa e trascurata - al contrario della fede e della speranza, tanto chiare e d'uso tanto comune, è indispensabile alla fede e alla speranza stesse. Infatti la carità è pensabile anche di per sé: la fede e la speranza sono impensabili senza la carità: e non solo impensabili, ma mostruose. Quelle del Nazismo (e quindi di un intero popolo) erano fede e speranza senza carità. Lo stesso si dica per la Chiesa clericale.

Insomma il potere - qualunque potere - ha bisogno dell'alibi della fede e della speranza. Non ha affatto bisogno dell'alibi della carità.

$[\ldots]$

Lo scisma verrebbe dunque a dividere la Chiesa Cattolica in due tronconi: nel primo resterebbero solo la fede e la speranza, cioè le due informi e cieche forze del potere; nel secondo resterebbero la fede e la speranza con la carità. (Pasolini, I995, p. 54)

Significativo punto di contatto si può ravvisare a distanza di sedici anni con un altro intellettuale per molti versi affine a Pasolini (ma per molti altri agli antipodi), preoccupato anch'egli della presenza del sacro nel mondo contemporaneo: si tratta di Giovanni Testori che nel I99I prende a tradurre in versi la Prima lettera ai Corinti di san Paolo in cui grande importanza riveste (per dichiarazione dell'autore stesso) la parole chiave carità:

6. Pasolini dichiara in versi: "Tuttavia, sia pure a parole, non si è mai dimenticata, / essa Chiesa, della Carità. Anzi, ci son esempi (tra i piccoli: / no, no, non certo qui in Vaticano) di pura carità. // La Chiesa vi contribuì dunque perché? Perché essa è, diletti figli, / istituzione!! / [...] // In quanto istituzione la Chiesa ha così contribuito / a sopprimere di fatto, la carità nel comportamento.” (Pasolini, I97I, pp. I7-I8) 
l'interesse suo

del tutto ignora;

non s'irrita;

il male non soppesa;

dell'ingiustizia non gioisce;

della giustizia è felice.

Tutto la carità

su di sé carca;

tutto la carità

sopporta;

(Testori, I991, pp. 89-90).

... e sola è portatrice di pietà e perdono. A questo proposito proprio nello stesso 1991 Testori andava scrivendo una pièce teatrale incentrata sul mito degli Atridi che traduceva in veste drammaturgica questo fondamentale aspetto della predicazione paolina: infatti Oreste, l'eroe della vendetta per antonomasia, rifiuta con un colpo di scena il ruolo assegnatogli da secoli di tradizione in nome della carità e del perdono diventando in questa negazione di se stesso sdisOrè (Testori, I99Ib).

Per concludere: se Testori sceglie un filtro classico per veicolare l'accanita denuncia della scomparsa del sacro nel contemporaneo (con esso del valore fondante della carità) per donarcene letterariamente una nuova sconcertante epifania, Pasolini ha scelto invece la figura di san Paolo, l'apostolo la cui predicazione controcorrente ha avuto spesso il potere di provocare e scandalizzare, il Santo che predica nel deserto di un'epoca in piena crisi sociale e ideologica con il quale Pasolini doveva sentirsi per molti, troppi versi affine. Non importa se la sua predicazione è una goccia in un deserto, non importa se non riesce a penetrare nell'indifferenza dei nuovi adepti dei consumi che guardano distratti il martirio del Santo in mezzo al traffico della periferia di una grande città della modernità: l'importante è che "in quel mondo di acciaio e di cemento è risuonata (o è tornata a risuonare) la parola di 'Dio'” (Pasolini, I977, p. I2)7.

7. Un esperimento sulla comparsa del sacro nel deserto della modernità, contemporaneo alla prima realizzazione dell'abbozzo sul San Paolo e di cui questo si può ritenere la prosecuzione, è rappresentata dal racconto cinematografico Teorema (poi effettivamente diventato film) che rispetto al microcosmo sociale descritto nella vicenda di Paolo, prende invece in considerazione il microcosmo di una famiglia della ricca borghesia milanese: il sacro, rappresentato in questo caso dall'irresistibile ospite-messaggero divino, si insinua nella coscienza borghese della famiglia e diventa un pericolo per le convenzioni e gli ipocriti conformismi. È lo scandalo attraverso il quale ogni membro della famiglia deve constatare il crollo di tutte le certezze in precedenza garanti dell'identità personale e sociale fino a scoprirsi diverso, inautentico.

A testimonianza dell'affinità fra i due lavori vanno chiari rimandi tematici come ad esempio la descrizione in Teorema dell'esperienza mistica vissuta da Paolo sulla via di Damasco in analogia con la folgorazione religiosa del capofamiglia (non a caso di nome Paolo) che finisce per spogliarsi di ogni bene per percorrere le strade della Milano moderna e industriale, nell'attesa di sentire ancora una volta lo sconvolgente appello del sacro 


\section{Bibliografia}

Conti Calabrese G. (1994), Pasolini e il sacro, Milano, Jaca Book, 1994.

De Giusti L. (i974), Pasolini P.P., "La perdita della realtà e il cinema inintegrabile" (conversazione con G. Bachmann del I3 settembre 1974), in id., Cinema in forma di poesia, a cura di L. De Giusti, Pordenone, Cinema Zero, 1979, pp. I56-I57.

Duflot J. (1970), Entretiens avec Pier Paolo Pasolini [1969], Paris, Belfond, 1970. Tr. it. P.P. Pasolini, Il sogno del centauro, a cura di J. Duflot, Roma, Editori Riuniti, 1983.

Eliade M. (1948), Traité d'histoire des religions, Paris, Payot, 1948. Tr. it.

Trattato di storia delle religioni, Torino, Einaudi, 1954.

Pasolini P. P. (I97I), Trasumanar e organizzar, Milano, Garzanti, I97I.

Pasolini P. P. (1975), Le Poesie, Milano, Garzanti, 1975.

Pasolini P. P. (1975b), Scritti Corsari, Milano, Garzanti, 1975.

Pasolini P. P. (1977), San Paolo, Torino, Einaudi, 1977.

Pasolini P. P. (1995), Il Caos, Roma, Editori Riuniti, 1995.

Pasolini P. P. (200I), Romanzi e Racconti 1962-1975, Milano, Mondadori, $200 I$.

Testori G. (1991), Traduzione della prima lettera ai Corinzi, Milano, Longanesi, I99I.

Testori G. (I99ib), sdisOrè, Milano, Longanesi, I99I.

(cfr. Pasolini, 200I, pp. 963-964; la sezione 27 di Teorema, Gli Ebrei si incamminarono verso il deserto, è tutta incentrata sulla figura di Paolo). L'analogia si fa ancora più esplicita attraverso le parole stesse del protagonista che performativamente commenta il suo cammino di "folgorato" per le strade della città (cfr. Pasolini, 200I, pp. 1053-1054). 
